\title{
Study of Concepts of Parallel Kinematics Machines for Advanced Manufacturing
}

\begin{abstract}
M. Valášek, V. Bauma, Z. Šika
This paper deals with possible new concepts for machine tools based on parallel kinematics for advanced manufacturing. Parallel kinematics machines (PKM) enable the mechanical properties of manufacturing machines to be improved. This has been proven by several new machine tool concepts. However, this potential can be and must be increased by applying the principle of redundant actuation. This paper deals with the extension of the concepts of redundantly actuated parallel kinematics structures for five-sided five-axis machine tools and for a free-forming sheet metal forming machine. The design principles of previous successful PKMs are summarized and new concepts are proposed. The most important requirement criteria are summarized. The proposed concepts are qualitatively and initially quantitatively evaluated according to these criteria.
\end{abstract}

Keywords: parallel kinematics, machine tools, metal forming machines, conceptual design.

\section{Introduction}

Parallel kinematics machines (PKM) represent a new concept in the design of machine tools [6, 7]. This concept has been investigated since the early 1990s. Parallel kinematics machines (PKM) enable the mechanical properties of manufacturing machines, especially the dynamics, to be improved. This has been proven by several new machine tool concepts. However, few PKMs have been successful on the market, due to new design problems resulting from the parallel kinematics concept. This leads to reduced workspace and stiffness. However, these problems can be removed and the potential of PKM can be be increased by applying the principle of redundant actuation [1]. This paper deals with the extension of the concepts of redundantly actuated parallel kinematics structures for five-sided five-axis machine tools and for a free-forming sheet metal forming machine. These concepts can be divided into full, hybrid and modular PKMs.

\section{Full parallel kinematics machines}

In full parallel kinematics machines, all DOFs are realized by the motion of the platform, and the platform is suspended by parallel links with drives on the frame. An example of spatial redundant full parallel kinematics is Octapod [2] (Fig. 1) corresponding to the traditional Hexapod. Eight links were selected for Octapod, instead of six links of Hexapod. In the initial concept, both the frame and the platform were cubes (Fig. 2a). The links connecting the vertices of the cubes are translational actuators, which are connected to the frame and the platform by spherical joints. The links have variable lengths. The first design problem was that the initial concept with a cubic platform was singular in the whole workspace. It was necessary to generate a modified platform concept. The modified platform consists of skew mutually rotated rectangles (Fig. 2b), and parameter optimisation of its dimensions was provided. This PKM is non-singular, and has good dexterity in the whole workspace.

The workspace is in principle equal to the whole cube of the frame without the boundary layer of the platform thickness. The orientation capability of the platform is good (more than +- 90 degrees have been achieved). If the platform is just a fraction of the frame cube (e.g. 1:5), then the ratio between the workspace and the machine space is much better than for other parallel kinematics concepts.

However, these properties pose quite large demands on the angular extent of the spherical joints, and these requirements have led to a special design of the new spherical joints [3]. This involves the possibility of five-sided machining. Five-sided machining means that a machine part (e.g. a cube) is fixed within the workspace and all five free sides of this cube are machined without any other fixing. Severe problems are posed by collisions. In our case this is influenced by the relative size of the platform, the position of the fixing table within the workspace and the resulting cube being five-side machinable. These parameters were thoroughly optimised, with large computational demands. The result is that a cube with the size of $25 \%$ of the Octapod frame can be really five-side machined.

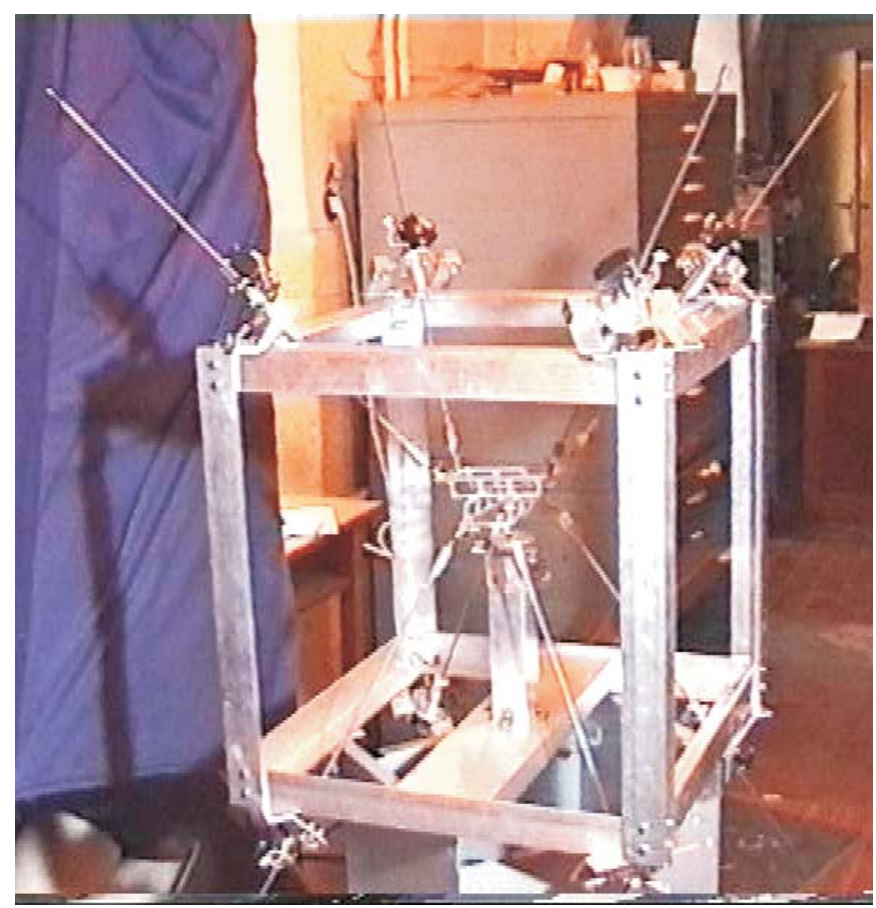

Fig. 1: Laboratory model of Octapod 


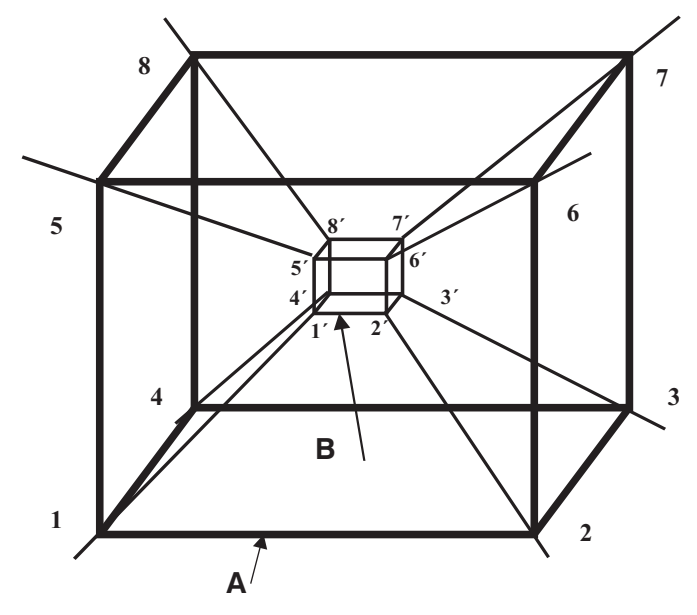

(a)

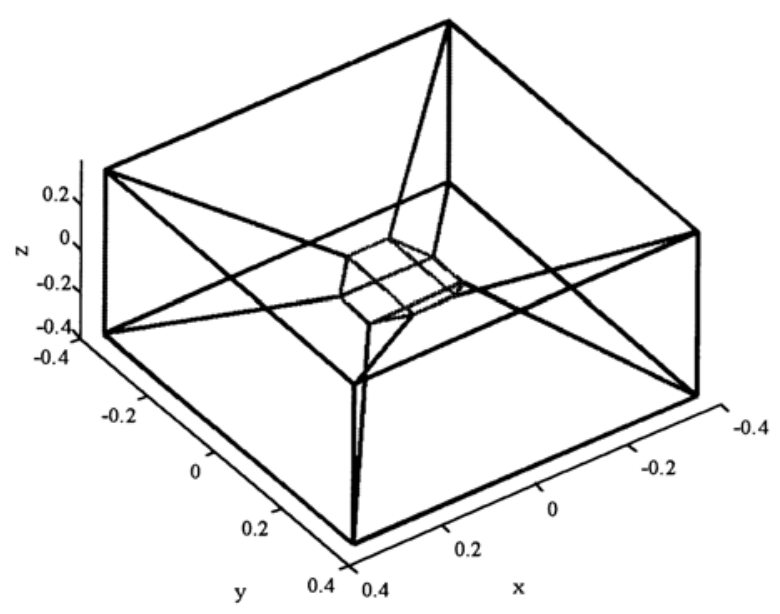

(b)

Fig. 2: The initial (a) and final (b) structure of OCTAPOD spatial redundant parallel kinematics

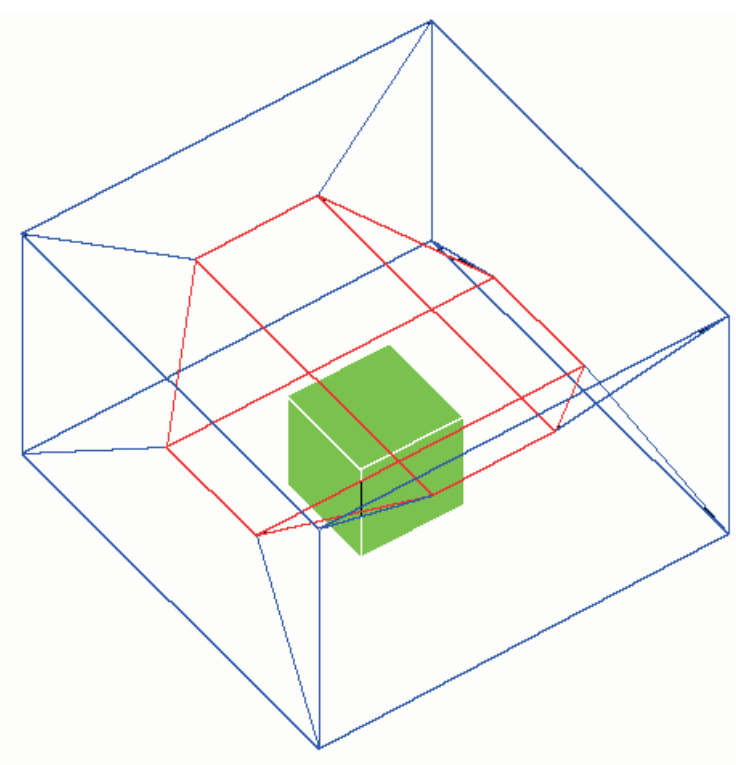

Fig. 3: Octapod variant for five-sided machining

Another example of a spatial full parallel kinematics machine is Octaslide [4]. It is a redundant version of Hexaslide.
It is a parallel kinematics machine the links of which have sliding actuators. The basic concept of such a structure is shown in Fig. 4. The platform is suspended on 8 links with actuators, whereas Hexaslide or Pentaslide are suspended on only 6 or 5 links.

The concept of redundant actuation completely removes the singularities from the workspace. The stiffness in the whole workspace was increased in maximum values by $65-74 \%$, and in average values by $43-54 \%$ compared to Hexaslide. Then the Octaslide concept was optimised as a machine tool for five-axis machining. The Hexaslide and Octaslide concepts were simultaneously intensively optimized. The result is that Octaslide is superior in all mechanical properties. The resulting concept of Octaslide is an asymmetric structure (Fig. 5), both in connections of the links with the frame and in the conical form of the platform. The increased orientation angle is $\pm 33^{\circ}$.

\section{Hybrid parallel kinematics machines}

In hybrid parallel kinematics machines, the DOFs of the machine are split into two parts, at least one of which is realized by the parallel kinematics concept. There are two groups

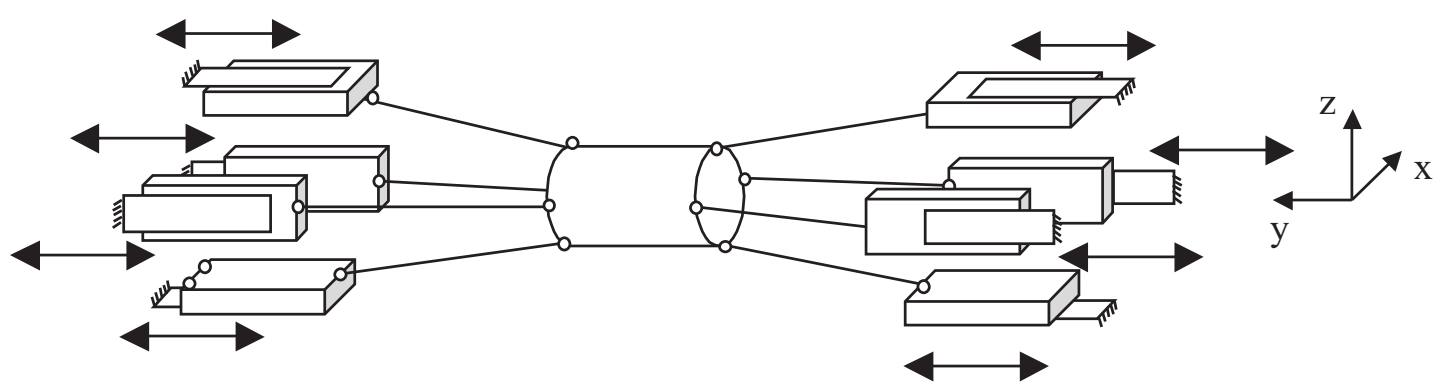

Fig. 4: Kinematical concept of Octaslide 


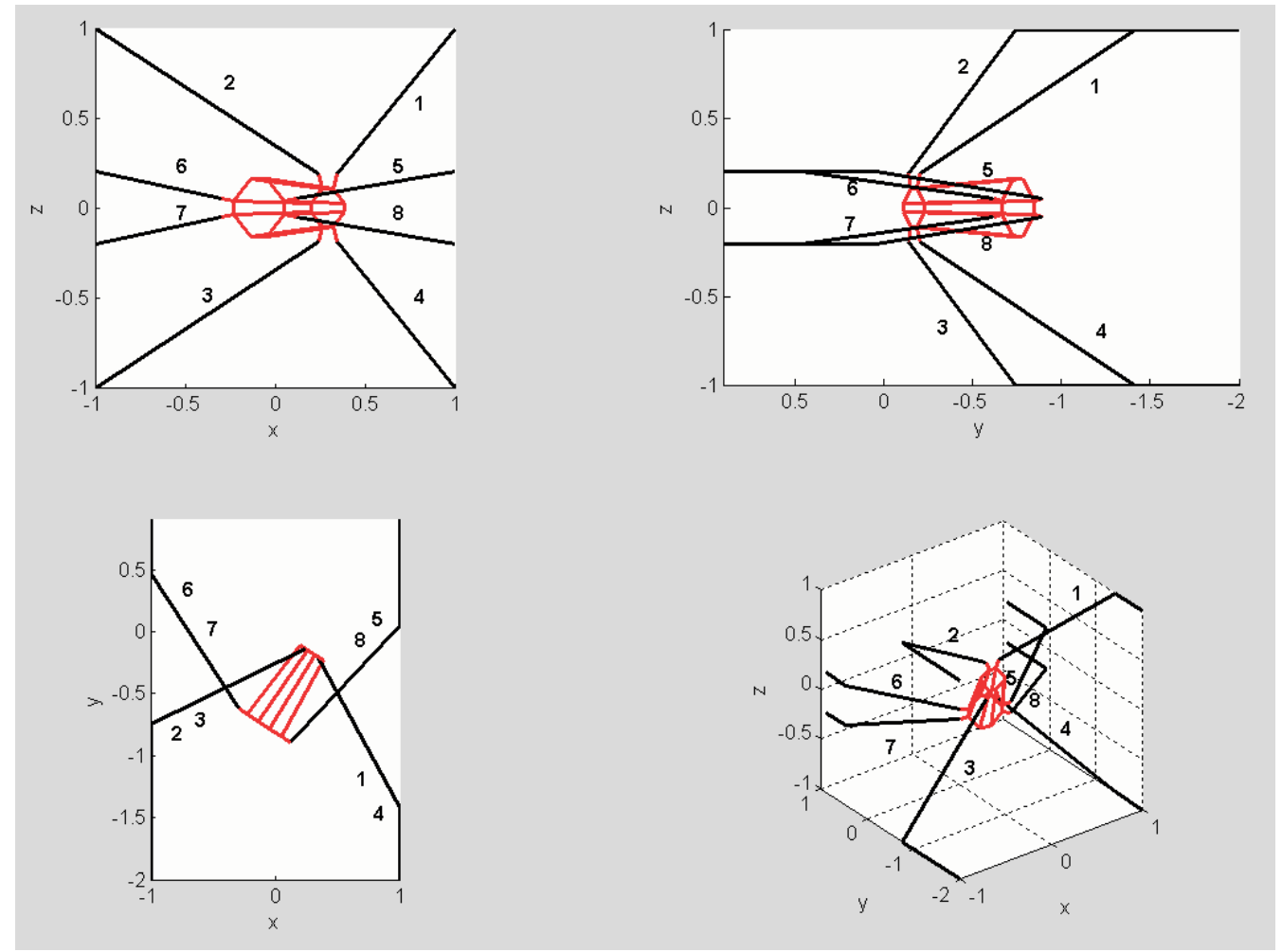

Fig. 5: Resulting variant of Octaslide with a conical platform

of concepts for five-axis/five-sided machining. They are based on dividing the required 5 DOFs into two parts, with $3+2$ DOFs or $4+1$ DOFs.

One group of hybrid PKMs is based on a planar parallel kinematics mechanism that realizes 3 DOFs in a large range, and this mechanism is combined with a translational table that can also rotate (two further DOFs). An example is shown in Fig. 6. An important feature is redundant actuation, which enables such a large range of motions to be realized.

Another other group of hybrid PKMs is based on a parallel kinematics mechanism that realizes spatial translational Cartesian motions and one rotation of the spindle (4 DOFs),

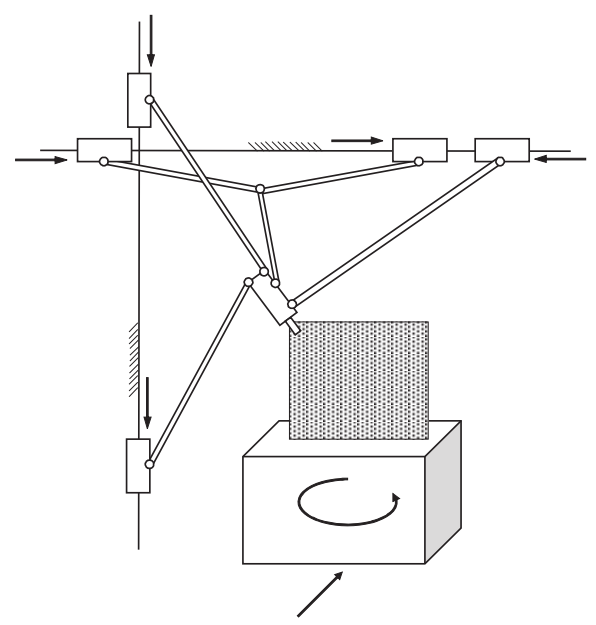

Fig. 6: An example of a PKM based on a redundant planar parallel mechanism with a large range of motions and this mechanism is combined with a rotary table (1 DOF). An example is shown in Fig. 7. It uses modifications of the advantageous module of Trijoint [5] (Fig. 8) as the portal horizontal mechanisms for the motion of the quill carrier, for quill travelling and even for spindle rotation.

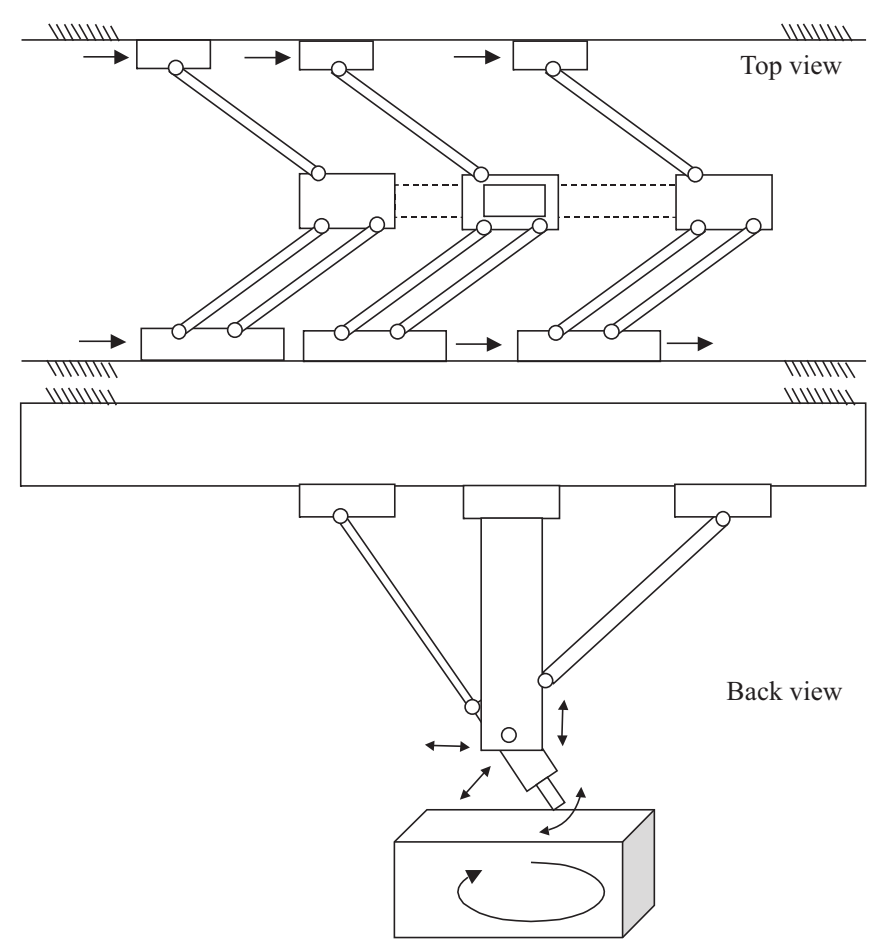

Fig. 7: An example of a PKM based on a portal mechanism with redundant modules 


\section{Modular parallel kinematics machines}

Modular parallel kinematics machines form a special group of hybrid PKMs that are based on PKM for spatial translational Cartesian motions combined with a swivel head for additional orientation of the spindle. They use the increased stiffness and dynamics of both parts - basic PKM for translational motions and the swivel head for orientation. If the swivel head is realized by a PKM, it solves the great problem of traditional swivel heads on composed rotational axes - that the head cannot always move directly to the required orientation. The basic PKM can implement all three Cartesian motions (3 DOFs) and the swivel head has only 2
DOFs (2 orientation angles), or the basic PKM can implement only two Cartesian motions (2 DOFs) and the swivel head has 3 DOFs (2 orientation angles and translation, as in the case of current parallel swivel heads).

This modular solution has many possible variants. The basic PKM can be based on Sliding Delta [2] (Redundant Uran) with three Cartesian motions (Fig. 9), or on Trijoint (Fig. 8) or Sliding Star [2], with two Cartesian motions (Fig. 10). The swivel head is mounted on the platform of the basic PKM. The swivel head can be based on a traditional Cardan mechanism or on parallel mechanisms. A redundantly actuated parallel swivel head with 3 DOFs (2 rotations, 1 translations) with increased stiffness and dynamics is shown in Fig. 11.
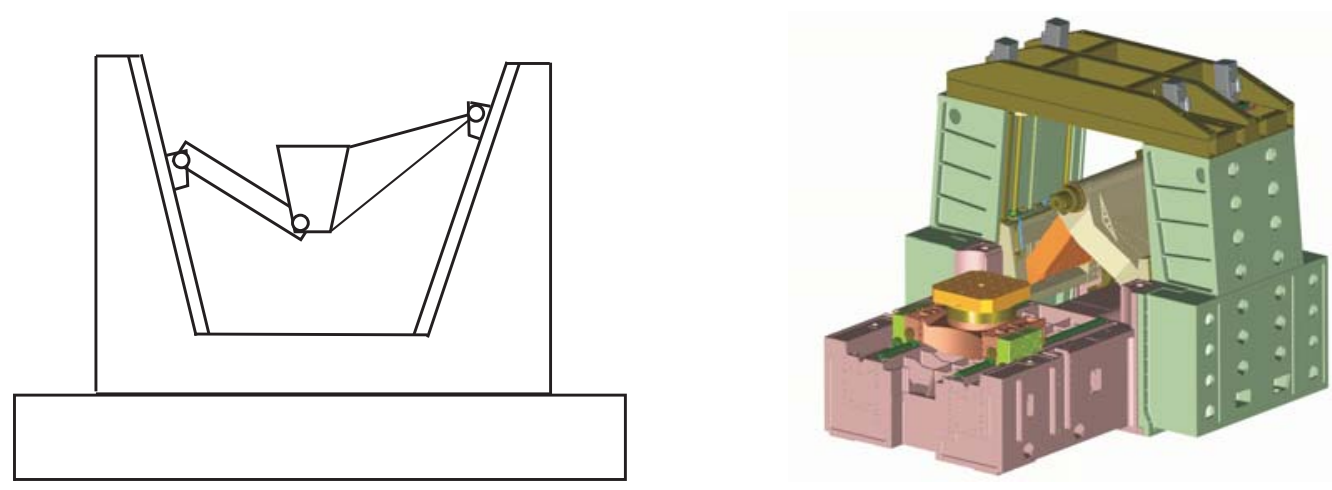

Fig. 8: Kinematical concept and design of Trijoint
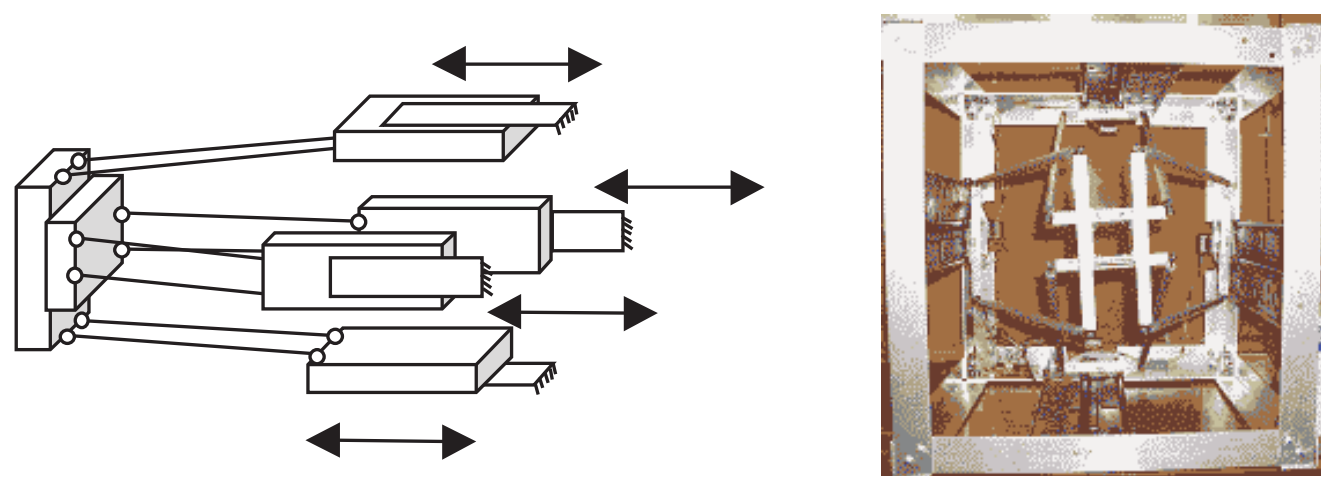

Fig. 9: Kinematical concept and laboratory model of Sliding Delta (redundant Uran)
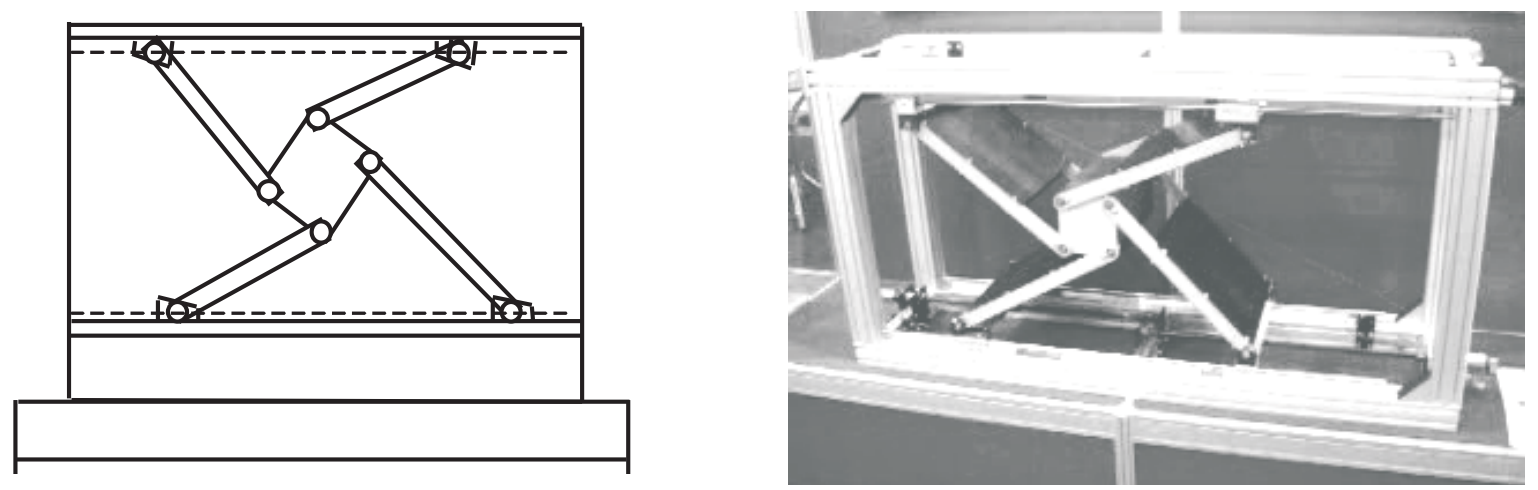

Fig. 10: Kinematical concept and laboratory model of Sliding Star 


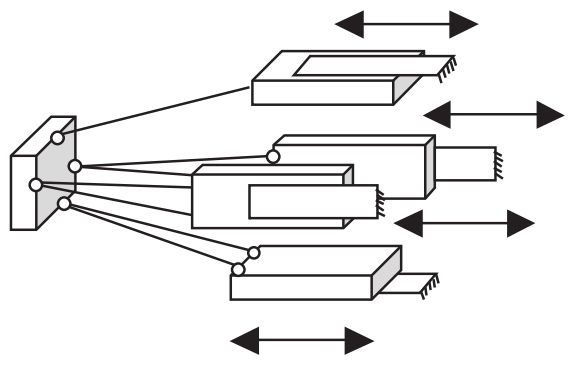

Fig. 11: Redundantly actuated swivel head

\section{Parallel kinematics metal forming machines}

There is only one example of the application of parallel kinematics for metal forming machines. This is Hexabend, produced by IWU FhG Chemnitz - PKM for free forming of tubes and similar rod profiles. Hexabend consists of a traditional feed mechanism for travelling the tube, together with Hexapod for bending the tube in any direction. This concept can be extended for metal forming of sheets (Fig. 12). One parallel kinematics mechanism acts as the holder and manipulator, and the other acts as the universal forming tool. It can have planar and spatial variants.

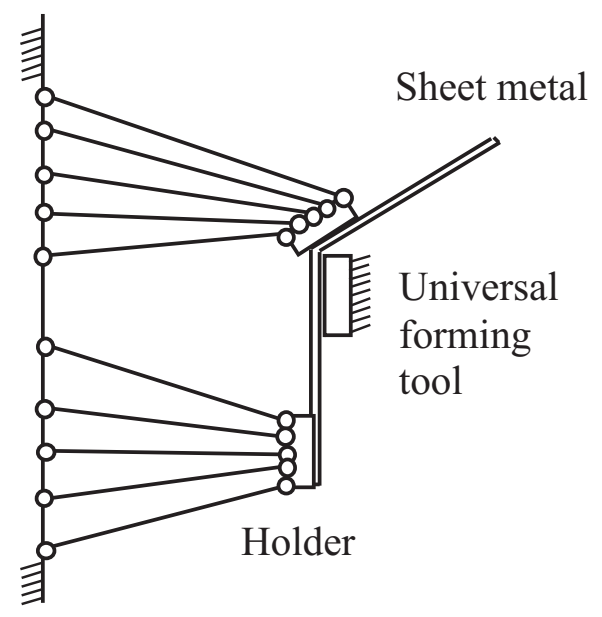

Fig. 12: Sheet metal forming by PKM

\section{Conclusion}

This paper describes different ways of extending PKMs for five-axis/ five-sided machining and for metal forming machines. The initial concept of parallel kinematics was oriented towards such applications, especially five-sided - five-axis machining, but design problems leading to limited workspace prevented the development of PKM with these applications. Current progress in designing PKMs without these design problems enables PKM to be proposed again for such de- manding applications. The paper summarizes several ways for designing such machines.

\section{Acknowledgment}

The authors appreciate the kind support by MSMT grant J04/98:212200008 "Development of methods and tools of integrated mechanical engineering”.

\section{References}

[1] Valasek, M., Sika, Z.: "Redundantly Actuated Parallel Kinematics-New Concept for Machine Tools.” In: Proc. of $1^{\text {st }}$ IFAC-Conference on Mechatronic Systems, Darmstadt 2000, p. 241-246.

[2] Valasek, M., Bauma, V., Sika, Z., Vampola, T.: "Redundantly Actuated Parallel Structures - Principle, Examples, Advantages." In: Neugebauer, R., (ed.): Development Methods and Application Experience of Parallel Kinematics, PKS 2002, IWU FhG, Chemnitz 2002, p. 993-1009.

[3] Valasek, M., Sulamanidze, D., Bauma, V.: "Spherical Joint with Increased Mobility for Octapod." In: Neugebauer, R., (ed.): Development Methods and Application Experience of Parallel Kinematics, PKS 2002, IWU FhG, Chemnitz 2002, p. 285-294.

[4] Bauma, V., Valasek, M., Sika, Z.: "Design and Properties of Octaslide Redundant Parallel Kinematics." In: Proc. of International Conference on Advanced Engineering Design AED 03, CTU, Prague 2003, p. C3.6/1-8

[5] Petru, F., Valasek, M.: "Concept, Design and Evaluated Properties of TRIJOINT 900H.” In: Neugebauer, R. (ed.): Proc. of PKS 2004 Parallel Kinematics Seminar, Chemnitz 2004.

[6] Neugebauer, R., (ed.): "Development Methods and Application Experience of Parallel Kinematics.” PKS 2002, IWU FhG, Chemnitz 2002.

[7] Neugebauer, R., (ed.): "Parallel Kinematic Seminar PKS 2004.” IWU FhG, Chemnitz 2004.

Prof. Michael Valášek, DrSc. valasek@fsik.cvut.cz

Ing. Václav Bauma, CSc. steinb@fsik.cvut.cz

Ing. Zbyněk Šika, PhD.

sika@fsik.cvut.cz

Department of Mechanics

Czech Technical University in Prague Faculty of Mechanical Engineering

Karlovo nám. 13

12135 Praha 2, Czech Republic 